SELECCIONES MATEMÁTICAS
Universidad Nacional de Trujillo
ISSN: $2411-1783$ (Online)
Vol. $05(02): 204-211(2018)$

\title{
Minimización y Maximización de Funciones Casisupermodulares
}

\section{Minization and Maximization of Quasi-Supermodular Functions}

\author{
Nelson Omar Aragonés Salazar*
}

Received, Oct. 05, 2018

Accepted, Nov. 30, 2018

DOI: http://dx.doi.org/10.17268/sel.mat.2018.02.07

\section{Resumen}

En este artículo se presentan algunas propiedades de la función casisupermodular y se demuestran principios de descarte para solucionar el problema de la minimización y maximización de este tipo de función definida en la familia de subconjuntos de un conjunto finito dado (retículo booleano finito); estos principios se generalizan, para el caso de la minimización, hasta un retículo finito relativamente complementado. Este trabajo extiende algunos resultados obtenidos por V.R. Jachatúrov y R.V. Jachatúrov para el caso de funciones supermodulares.

Palabras clave. Optimización combinatoria, función casisupermodular, función supermodular, retículo finito, retículo booleano.

\begin{abstract}
This article presents some properties of the casisupermodular function and demonstrates principles of discarding to solve the problem of minimization and maximization of this type of function defined in the family of subsets of a given finite set (finite boolean lattice); these principles are generalized, in the case of minimization, to a relatively complemented finite lattice. This work extends some results obtained by V.R. Khachaturov and R.V. Khachaturov for the case of supermodular functions.
\end{abstract}

Keywords. Combinatorial optimization, quasi-supermodular function, supermodular function, finite lattice, boolean lattice.

\section{Introducción.}

El presente trabajo expone algunas propiedades de las funciones casisupermodulares, así como principios de descarte que constituyen el fundamento matemático para la búsqueda de los mínimos y máximos de una función casisupermodular (c-supermodular) definida en la familia de subconjuntos de un conjunto finito dado (retículo booleano), así como la generalización de estos principios, hasta un retículo relativamente complementado, para el caso de la minimización de una función c-supermodular.

Además del desarrollo de esquemas generales de programación combinatoria es importante la creación de métodos de solución para clases específicas de problemas.

\footnotetext{
${ }^{*}$ Facultad de Ciencias Físicas y Matemáticas, Universidad Nacional de Trujillo, Av. Juan Pablo II, s/n, Trujillo-Perú (omararagones@yahoo. com).

This work is licensed under the Creative Commons Attribution-NoComercial-ShareAlike 4.0.
} 
Uno de los avances más interesantes y originales en esta dirección lo conforman los trabajos de V.P. Cherenin, V.R. Jachatúrov y R.V. Jachatúrov en los que se desarrolla el "método de cálculos sucesivos" para encontrar los mínimos y máximos de una función supermodular definida en todos los subconjuntos de un conjunto finito dado, así como su generalización para el caso de la optimización de funciones supermodulares definidas en diferentes tipos de retículos finitos (retículos bboleano, con complemento relativo, de particiones, de subespacion vectoriales, geométricas, de producto directo de cadenas, de cubos) [4] [5] [6] [7] [8] [9] . Es interesante indicar que el primer trabajo sobre este método y su aplicación en la solución de un tipo de problema aplicado (formación del plan de formación de trenes) se debe a Cherenin y data de 1948.

Los resultados presentados en este trabajo extienden algunos de los conseguidos por V.P. Cherenin, V.R. Jachatúrov y R.V. Jachatúrov para funciones supermodulares [6] [8] [1].

\subsection{Funciones supermodulares.}

Sea el conjunto finito $I=\{1,2, \ldots, m\}$ y $f$ una función definida en $\Omega=2^{I}$, la clase de todos los subconjuntos de $I$

Definición 1. La función $f$ se denomina supermodular si para cualesquiera $\omega_{1}, \omega_{2} \in \Omega$ tiene lugar la desigualdad siguiente

$$
f\left(\omega_{1}\right)+f\left(\omega_{2}\right) \leq f\left(\omega_{1} \cup \omega_{2}\right)+f\left(\omega_{1} \cap \omega_{2}\right)
$$

\subsection{Minimización de funciones supermodulares.}

Para la función supermodular $f$ considérese el siguiente problema.

Determinar $\alpha \in \Omega$ tal que

$$
f(\alpha)=\min _{\omega \in \Omega} f(\omega)
$$

En [6] [8] [9] [1] se presentan tres principios de descarte, que son los siguientes:

\section{Primer principio de descarte.}

Sea $f$ supermodular y para $\omega_{1} \subset \omega_{2}$ se tenga que $f\left(\omega_{1}\right)<f\left(\omega_{2}\right)$, entonces se pueden descartar todos los $\omega$ tales que $\omega_{2} \subset \omega$.

\section{Segundo principio de descarte.}

Sea $f$ supermodular y para $\omega_{1} \subset \omega_{2}$ se tenga que $f\left(\omega_{1}\right)>f\left(\omega_{2}\right)$, entonces se pueden descartar todos los $\omega$ tales que $\omega \subset \omega_{1}$.

Definamos para $i \in \omega_{2} \backslash \omega_{1}$

$$
\begin{gathered}
\Delta_{1}(i)=\left\{\begin{array}{cl}
f\left(\omega_{1}\right)-f\left(\omega_{1} \cup\{i\}\right), & \text { si } f\left(\omega_{1}\right)-f\left(\omega_{1} \cup\{i\}\right) \geq 0, \\
0 \quad & \text { si } f\left(\omega_{1}\right)-f\left(\omega_{1} \cup\{i\}\right)<0 .
\end{array}\right. \\
L_{1}\left(\omega_{1}, \omega_{2}\right):=f\left(\omega_{1}\right)-\sum_{i \in \omega_{2} \backslash \omega_{1}} \Delta_{1}(i) \\
\Delta_{2}(i)=\left\{\begin{array}{cl}
f\left(\omega_{2}\right)-f\left(\omega_{2} \backslash\{i\}\right), & \text { si } f\left(\omega_{2}\right)-f\left(\omega_{2} \backslash\{i\}\right) \geq 0, \\
0 \quad & \text { si } f\left(\omega_{2}\right)-f\left(\omega_{2} \backslash\{i\}\right)<0 .
\end{array}\right.
\end{gathered}
$$




$$
L_{2}\left(\omega_{1}, \omega_{2}\right):=f\left(\omega_{2}\right)-\sum_{i \in \omega_{2} \backslash \omega_{1}} \Delta_{2}(i) .
$$

\section{Tercer principio de descarte.}

Sea $f$ supermodular y para $\omega_{1} \subset \omega_{2}$ se tenga que $L_{1}\left(\omega_{1}, \omega_{2}\right)>f(\widetilde{\alpha})$ ó $L_{2}\left(\omega_{1}, \omega_{2}\right)>f(\widetilde{\alpha})$ donde $\widetilde{\alpha}$ es la "mejor"variante conocida hasta un determinado momento, entonces se descartan todas las variantes $\omega$ tales que $\omega_{1} \subset \omega \subset \omega_{2}$, pues $f(\omega)>f(\alpha)$.

\subsection{Maximización de funciones supermodulares.}

Sea la función supermodular $f$, considérese el siguiente problema.

Determinar $\theta \in \Omega$ tal que

$$
f(\theta)=\operatorname{máx}_{\omega \in \Omega} f(\omega)
$$

Se dice que $f$ tiene en $\theta_{u} \in \Omega$ un máximo local si: $f\left(\theta_{u}\right) \geq f\left(\theta_{u} \cup\{i\}\right)$ para todo $i \in I \backslash \theta_{u}$ y $f\left(\theta_{u}\right) \geq$ $f\left(\theta_{u} \backslash\{i\}\right)$ para todo $i \in \theta_{u}$. Es evidente que todo máximo global es máximo local.

Denotemos por $\theta_{u}^{\prime}$ a algún máximo local de $f(\omega)$ en $\omega_{1} \subset \omega \subset \omega_{2}$, en [4] [8] se presentan tres principios de descarte, que son los siguientes:

\section{Primer principio de descarte.}

Si para algún $i_{0} \in \omega_{2} \backslash \omega_{1}$ resulta que $f\left(\omega_{1}\right)<f\left(\omega_{1} \cup\left\{i_{0}\right\}\right)$, entonces $i_{0} \in \theta_{u}^{\prime}$; es decir, $i_{0}$ se encuentra en todos los máximos locales $\theta_{u}^{\prime}$.

\section{Segundo principio de descarte.}

Si para algún $i_{0} \in \omega_{2} \backslash \omega_{1}$ resulta que $f\left(\omega_{2}\right)<f\left(\omega_{2} \backslash\left\{i_{0}\right\}\right)$, entonces $i_{0} \notin \theta_{u}^{\prime}$.

Definamos para $\omega_{1}, \omega_{2}$

$$
\begin{aligned}
& P_{1}\left(\omega_{1}, \omega_{2}\right):=f\left(\omega_{2}\right)+\sum_{i \in \omega_{2} \backslash \omega_{1}}\left[f\left(\omega_{1}\right)-f\left(\omega_{1} \cup i\right)\right], \\
& P_{2}\left(\omega_{1}, \omega_{2}\right):=f\left(\omega_{1}\right)+\sum_{i \in \omega_{2} \backslash \omega_{1}}\left[f\left(\omega_{2}\right)-f\left(\omega_{2} \backslash i\right)\right] .
\end{aligned}
$$

\section{Tercer principio de descarte.}

Sea $f$ supermodular y para $\omega_{1} \subset \omega_{2}$ se tenga que $P_{1}\left(\omega_{1}, \omega_{2}\right)<f(\widetilde{\theta})$ ó $P_{2}\left(\omega_{1}, \omega_{2}\right)<f(\widetilde{\theta})$ donde $\widetilde{\theta}$ es la "mejor"variante conocida hasta un determinado momento, entonces se descartan todas las variantes $\omega$ tales que $\omega_{1} \subset \omega \subset \omega_{2}$, pues $f(\omega)<f(\theta)$.

Estos principios de descarte permiten reducir el espacio de búsqueda y así evitar la búsqueda exhaustiva. Por lo general el orden del volumen de selección basado en los principios de descarte expuestos no supera $m^{3}$, que es significativamente menor que el volumen de la selección completa $2^{m}$ [8].

En los trabajos de Cherenin y Cherenin y Jachaturov se presentan las formulaciones de una serie de problemas aplicados que pueden ser exitosamente resueltos con el método de cálculos sucesivos basado en los principios de descarte expuestos en [4] [6] [8]. Algunos de estos problemas son el modelo de localización de empresas considerando el capital de inversión para su construcción así como los gastos de transporte [6] y el de optimización del agrupamiento de zonas de una región [4].

En lo que sigue se demostrará la validez de estos principios de descarte para el caso de funciones casisupermodulares. 


\section{Funciones casisupermodulares.}

Sean $I=\{1,2, \ldots, m\}, \Omega=2^{I}$ y $f: \Omega \rightarrow \mathbb{R}$.

Definición 2. Se dice que la función $f$ es casisupermodular (c-supermodular) si para cualesquiera $\omega_{1}, \omega_{2} \in \Omega$ tienen lugar las implicaciones siguientes:

1. $f\left(\omega_{1}\right) \geq f\left(\omega_{1} \cap \omega_{2}\right) \Rightarrow f\left(\omega_{1} \cup \omega_{2}\right) \geq f\left(\omega_{2}\right)$,

2. $f\left(\omega_{1}\right)>f\left(\omega_{1} \cap \omega_{2}\right) \Rightarrow f\left(\omega_{1} \cup \omega_{2}\right)>f\left(\omega_{2}\right)$.

Consideremos algunas propiedades de las funciones c-supermodulares [10].

\section{Propiedades.}

1. Si $f$ es c-supermodular, entonces tienen lugar las implicaciones siguientes:

(a) $f\left(\omega_{2}\right) \geq f\left(\omega_{1} \cup \omega_{2}\right) \Rightarrow f\left(\omega_{1} \cap \omega_{2}\right) \geq f\left(\omega_{1}\right)$,

(b) $f\left(\omega_{2}\right)>f\left(\omega_{1} \cup \omega_{2}\right) \Rightarrow f\left(\omega_{1} \cap \omega_{2}\right)>f\left(\omega_{1}\right)$.

Demostración:

En efecto, sea $f$ c-supermodular y $f\left(\omega_{2}\right) \geq f\left(\omega_{1} \cup \omega_{2}\right)$, entonces asumiendo que tiene lugar la desigualdad contraria $f\left(\omega_{1} \cap \omega_{2}\right)<f\left(\omega_{1}\right)$ se tendría, por c-supermodularidad, que $f\left(\omega_{1} \cup \omega_{2}\right)>f\left(\omega_{2}\right)$; de donde $f\left(\omega_{1} \cup \omega_{2}\right)>f\left(\omega_{2}\right) \geq f\left(\omega_{1} \cup \omega_{2}\right)$, lo que es absurdo, por lo tanto $f\left(\omega_{1} \cap \omega_{2}\right) \geq f\left(\omega_{1}\right)$.

En forma similar se demuestra que la implicación $f\left(\omega_{2}\right)>f\left(\omega_{1} \cup \omega_{2}\right) \Rightarrow f\left(\omega_{1} \cap \omega_{2}\right)>f\left(\omega_{1}\right)$ es válida.

2. Si $f$ es c-supermodular, entonces $\forall A \subset B \subset I, i \in I \backslash B$ tienen lugar las implicaciones siguientes:

(a) $f(A \cup i) \geq f(A) \Longrightarrow f(B \cup i) \geq f(B)$,

(b) $f(A \cup i)>f(A) \Longrightarrow f(B \cup i)>f(B)$.

Demostración:

En efecto, para $\omega_{1}=B, \omega_{2}=A \cup i$ se tiene $\omega_{1} \cup \omega_{2}=B \cup i, \omega_{1} \cap \omega_{2}=A$. Por la c-supermodularidad de $f$ se tiene

$$
f\left(\omega_{2}\right)=f(A \cup i) \geq f(A)=f\left(\omega_{1} \cap \omega_{2}\right) \Longrightarrow f(B \cup i)=f\left(\omega_{1} \cup \omega_{2}\right) \geq f\left(\omega_{1}\right)=f(B) .
$$

En forma similar se demuestra que la implicación $f(A \cup i)>f(A) \Longrightarrow f(B \cup i)>f(B)$ es válida. $\square$

Una propiedad característica de las funciones c-supermodulares es el sub-cruce simple (SCS).

Definición 3. Se dice que la función f satisface la propiedad SCS si $\forall A \subset B \subset I, i \in I \backslash B$ tienen lugar las implicaciones siguientes:

1. $f(B) \geq f(A) \Longrightarrow f(B \cup i) \geq f(A \cup i)$,

2. $f(B)>f(A) \Longrightarrow f(B \cup i)>f(A \cup i)$.

\section{Teorema 1.}

$f$ es c-supermodular $\Longleftrightarrow f$ satisface la propiedad SCS.

Demostración:

En efecto, sea $f$ c-supermodular. Para $A \subset B \subset I, i \in I \backslash B$ definamos $\omega_{1}=A \cup i, \omega_{2}=B$, por lo que $\omega_{1} \cup \omega_{2}=B \cup i, \omega_{1} \cap \omega_{2}=A$. Entonces, si

$$
f\left(\omega_{2}\right)=f(B) \geq f(A)=f\left(\omega_{1} \cap \omega_{2}\right)
$$

por c-supermodularidad se tiene

$$
f\left(\omega_{1} \cup \omega_{2}\right)=f(B \cup i) \geq f(A \cup i)=f\left(\omega_{1}\right) .
$$

En forma similar se tiene

$$
f(B)>f(A) \Longrightarrow f(B \cup i)>f(A \cup i) .
$$


Recíprocamente, si $f$ satisface la propiedad SCS, entonces para $\omega_{1}, \omega_{2} \subset I$ denotemos $\omega_{2} \backslash \omega_{1}=\left\{i_{1}, i_{2}, \ldots, i_{k}\right\}$. Por la propiedad SCS, si

$$
f\left(\omega_{1}\right) \geq f\left(\omega_{1} \cap \omega_{2}\right) \Longrightarrow f\left(\omega_{1} \cup i_{1}\right) \geq f\left(\left(\omega_{1} \cap \omega_{2}\right) \cup i_{1}\right) \Longrightarrow
$$

$f\left(\omega_{1} \cup i_{1} \cup i_{2}\right) \geq f\left(\left(\omega_{1} \cap \omega_{2}\right) \cup i_{1} \cup i_{2}\right) \Longrightarrow \cdots \Longrightarrow f\left(\omega_{1} \cup i_{1} \cup i_{2} \cup \cdots \cup i_{k}\right) \geq f\left(\left(\omega_{1} \cap \omega_{2}\right) \cup i_{1} \cup i_{2} \cdots \cup i_{k}\right)$,

es decir $f\left(\omega_{1} \cup \omega_{2}\right) \geq f\left(\omega_{2}\right)$.

En forma similar se demuestra que $f\left(\omega_{1}\right)>f\left(\omega_{1} \cap \omega_{2}\right) \Longrightarrow f\left(\omega_{1} \cup \omega_{2}\right)>f\left(\omega_{2}\right)$.

\section{Proposición 1.}

Toda función supermodular es c-supermodular.

Demostración:

En efecto, sea $f$ supermodular, entonces

$$
f\left(\omega_{1}\right)-f\left(\omega_{1} \cap \omega_{2}\right) \leq f\left(\omega_{1} \cup \omega_{2}\right)-f\left(\omega_{2}\right),
$$

por lo tanto, si $0 \leq f\left(\omega_{1}\right)-f\left(\omega_{1} \cap \omega_{2}\right) \Rightarrow 0 \leq f\left(\omega_{1} \cup \omega_{2}\right)-f\left(\omega_{2}\right)$.

Obviamente, si $0<f\left(\omega_{1}\right)-f\left(\omega_{1} \cap \omega_{2}\right) \Rightarrow 0<f\left(\omega_{1} \cup \omega_{2}\right)-f\left(\omega_{2}\right)$; por lo tanto $f$ es c-supermodular. $\square$

No toda función c-supermodular es supermodular. Para verificar esto consideremos el ejemplo siguiente.

Ejemplo 1. Sea $I=\{1,2\}$ y $\Omega=\{\emptyset,\{1\},\{2\}, I\}$. Definamos $f(\emptyset)=0, f(\{1\})=f(\{2\})=2, f(I)=3$. Para $i \in\{1,2\}$ se tiene $2=f(\{i\})>f(\{1\} \cap\{2\})=f(\emptyset)=0$ y $3=f(I)=f(\{1\} \cup\{2\})>f(\{i\})=2$, por lo que $f$ es c-supermodular, sin embargo

$$
4=f(\{1\})+f(\{2\})>f(\{1\} \cap\{2\})+f(\{1\} \cup\{2\})=f(\emptyset)+f(I)=3,
$$

por lo que $f$ no es supermodular.

\subsection{Minimización de funciones c-supermodulares.}

Sea $f$ c-supermodular, consideremos el siguiente problema extremal.

Determinar $\alpha \in \Omega$ tal que

$$
f(\alpha)=\min _{\omega \in \Omega} f(\omega)
$$

\section{Teorema 2 (Primer principio de descarte).}

Si para $\omega_{1} \subset \omega_{2} \subset I$ se tiene $f\left(\omega_{1}\right)<f\left(\omega_{2}\right)$, entonces pueden descartarse todas las $2^{n-\left|\omega_{2}\right|}$ variantes $\omega \supset \omega_{2}$ pues para ellas $f(\omega)>f(\alpha)$.

Demostración:

En efecto, sea que $f\left(\omega_{1}\right)<f\left(\omega_{2}\right), \omega \supset \omega_{2}$. Tomando $\delta=\omega_{2}, \gamma=\omega_{1} \cup\left(\omega \backslash \omega_{2},\right)$ entonces $\delta \cup \gamma=\omega$, $\delta \cap \gamma=\omega_{1}$

Así, por la c-supermodularidad de $f$ se tiene

$$
f(\delta \cap \gamma)=f\left(\omega_{1}\right)<f\left(\omega_{2}\right)=f(\delta) \quad \Longrightarrow \quad f(\gamma)<f(\delta \cup \gamma)=f(\omega)
$$

Por lo que $f(\alpha) \leq f(\gamma)<f(\omega)$.

El número de subconjuntos de $I$ que contienen a $\omega_{2}$ es $2^{n-\left|\omega_{2}\right|}$. $\square$

Teorema 3 (Segundo principio de descarte).

Si para $\omega_{1} \subset \omega_{2} \subset$ I se tiene $f\left(\omega_{1}\right)>f\left(\omega_{2}\right)$, entonces pueden descartarse todas las $2^{\left|\omega_{1}\right|}$ variantes $\omega \subset \omega_{1}$ pues para ellas $f(\omega)>f(\alpha)$.

Demostración: 
En efecto, sea que $f\left(\omega_{1}\right)>f\left(\omega_{2}\right)$ y $\omega \subset \omega_{1}$. Definamos $\delta=\omega_{1}, \gamma=\omega \cup\left(\omega_{2} \backslash \omega_{1},\right)$ entonces $\delta \cup \gamma=\omega_{2}$, $\delta \cap \gamma=\omega$.

Así, por propiedad,

$$
f(\delta)=f\left(\omega_{1}\right)>f\left(\omega_{2}\right)=f(\delta \cup \gamma) \quad \Longrightarrow \quad f(\omega)=f(\delta \cap \gamma)>f(\gamma),
$$

por lo que $f(\omega)>f(\gamma) \geq f(\alpha)$.

El número de subconjuntos de $I$ contenidos en $\omega_{1}$ es $2^{\left|\omega_{1}\right|}$.

\subsection{Maximización de funciones c-supermodulares.}

Sea $f$ c-supermodular, consideremos el siguiente problema extremal.

Determinar $\theta \in \Omega$ tal que

$$
f(\theta)=\operatorname{máx}_{\omega \in \Omega} f(\omega) .
$$

Teorema 4 (Primer principio de descarte).

Sean $\omega_{1} \subset \omega_{2} \subset I$. Si $\omega_{1} \subset \theta \subset \omega_{2}$ y para algún $i_{0} \in \omega_{2} \backslash \omega_{1}$ se tiene que $f\left(\omega_{1}\right)<f\left(\omega_{1} \cup i_{0}\right)$, entonces $i_{0} \in \theta$.

Demostración: En efecto, sea que $i_{0} \notin \theta$. Como $i_{0} \notin \omega_{1}$ y $\omega_{1} \subset \theta$, entonces $\left(\omega_{1} \cup i_{0}\right) \cap \theta=\omega_{1},\left(\omega_{1} \cup i_{0}\right) \cup \theta=$ $\theta \cup i_{0}$.

Así, por la c-supermodularidad de $f$ se tiene

$$
f\left(\omega_{1} \cup i_{0}\right)>f\left(\left(\omega_{1} \cup i_{0}\right) \cap \theta\right)=f\left(\omega_{1}\right) \quad \Longrightarrow \quad f\left(\left(\omega_{1} \cup i_{0}\right) \cup \theta\right)=f\left(\theta \cup i_{0}\right)>f(\theta) .
$$

Lo que es absurdo.

Teorema 5 (Segundo principio de descarte).

Sean $\omega_{1} \subset \omega_{2} \subset I$. Si $\omega_{1} \subset \theta \subset \omega_{2}$ y para algún $i_{0} \in \omega_{2} \backslash \omega_{1}$ se tiene que $f\left(\omega_{2}\right)<f\left(\omega_{2} \backslash i_{0}\right)$, entonces $i_{0} \notin \theta$.

Demostración: En efecto, sea que $i_{0} \in \theta$. Como $i_{0} \in \omega_{2}$ y $\theta \subset \omega_{2}$, entonces $\left(\omega_{2} \backslash i_{0}\right) \cap \theta=\theta \backslash i_{0}$, $\left(\omega_{2} \backslash i_{0}\right) \cup \theta=\omega_{2}$.

Así, por propiedad

$$
f\left(\omega_{2} \backslash i_{0}\right)>f\left(\left(\omega_{2} \backslash i_{0}\right) \cup \theta\right)=f\left(\omega_{2}\right) \quad \Longrightarrow \quad f\left(\left(\omega_{2} \backslash i_{0}\right) \cap \theta\right)=f\left(\theta \backslash i_{0}\right)>f(\theta) .
$$

Lo que es absurdo.

\section{Funciones c-supermodulares en retículos.}

Sea $\langle A ; \leq, \vee, \wedge\rangle$ un retículo, $a, b \in A, a \leq b$ y $[a, b]=\{x \in A: a \leq x \leq b\}$ un intervalo [3] [11]. El retículo $\langle A ; \leq, \vee, \wedge\rangle$ se denomina finito si $|A|<\infty$.

Definición 4. Se denomina complemento relativo del elemento $x \in[a, b]$ al elemento $x^{*} \in[a, b]$, tal que $x \wedge x^{*}=a y x \vee x^{*}=b$.

Definición 5. El retículo $\langle A ; \leq, \vee, \wedge\rangle$ se denomina retículo relativamente complementado si para todo $x \in A$ en todo intervalo que contiene a $x$ existe para este elemento complemento relativo $x^{*}$.

Sea $\Omega$ un retículo finito relativamente complementado [2], con orden parcial $\leq$.

Definición 6. La función $f: \Omega \rightarrow \mathbb{R}$ se denomina casisupermodular sobre $\Omega$ (c-supermodular) si para cualesquiera $\omega_{1}, \omega_{2} \in \Omega$ tienen lugar las implicaciones siguientes:

1. $f\left(\omega_{1}\right) \geq f\left(\omega_{1} \wedge \omega_{2}\right) \Rightarrow f\left(\omega_{1} \vee \omega_{2}\right) \geq f\left(\omega_{2}\right)$,

2. $f\left(\omega_{1}\right)>f\left(\omega_{1} \wedge \omega_{2}\right) \Rightarrow f\left(\omega_{1} \vee \omega_{2}\right)>f\left(\omega_{2}\right)$. 
Tiene lugar la siguiente propiedad.

Proposición 2. Si f es c-supermodular, entonces

$$
f\left(\omega_{2}\right) \geq f\left(\omega_{1} \vee \omega_{2}\right) \Rightarrow f\left(\omega_{1} \wedge \omega_{2}\right) \geq f\left(\omega_{1}\right) .
$$

Demostración:

En efecto, sea $f$ c-supermodular y $f\left(\omega_{2}\right) \geq f\left(\omega_{1} \vee \omega_{2}\right)$, entonces si $f\left(\omega_{1} \wedge \omega_{2}\right)<f\left(\omega_{1}\right)$ se tendría, por c-supermodularidad, que $f\left(\omega_{1} \vee \omega_{2}\right)>f\left(\omega_{2}\right)$; de donde $f\left(\omega_{1} \vee \omega_{2}\right)>f\left(\omega_{2}\right) \geq f\left(\omega_{1} \vee \omega_{2}\right)$, lo que es absurdo.

En forma similar se demuestra que si $f$ es c-supermodular, entonces

$$
f\left(\omega_{2}\right)>f\left(\omega_{1} \vee \omega_{2}\right) \Rightarrow f\left(\omega_{1} \wedge \omega_{2}\right)>f\left(\omega_{1}\right) .
$$

Definición 7. Se dice que $f$ es supermodular si para cualesquiera $\omega_{1}, \omega_{2} \in \Omega$ tiene lugar la desigualdad

$$
f\left(\omega_{1}\right)+f\left(\omega_{2}\right) \leq f\left(\omega_{1} \wedge \omega_{2}\right)+f\left(\omega_{1} \vee \omega_{2}\right) .
$$

Proposición 3. Toda función supermodular es c-supermodular.

Demostración:

En efecto, sea $f$ supermodular, entonces

$$
f\left(\omega_{1}\right)-f\left(\omega_{1} \wedge \omega_{2}\right) \leq f\left(\omega_{1} \vee \omega_{2}\right)-f\left(\omega_{2}\right),
$$

así, si $0 \leq f\left(\omega_{1}\right)-f\left(\omega_{1} \wedge \omega_{2}\right) \Rightarrow 0 \leq f\left(\omega_{1} \vee \omega_{2}\right)-f\left(\omega_{2}\right)$.

En forma similar, si $0<f\left(\omega_{1}\right)-f\left(\omega_{1} \wedge \omega_{2}\right) \Rightarrow 0<f\left(\omega_{1} \vee \omega_{2}\right)-f\left(\omega_{2}\right)$.

En general, no toda función c-supermodular es supermodular.

\subsection{Minimización en retículos.}

Sea $f$ c-supermodular, consideremos el siguiente problema extremal.

Determinar $\alpha \in \Omega$ tal que

$$
f(\alpha)=\min _{\omega \in \Omega} f(\omega)
$$

\section{Theorem 4.1 (Primer principio de descarte).}

Si para $\omega_{1}, \omega_{2} \in \Omega, \omega_{1} \leq \omega_{2}$ se tiene $f\left(\omega_{1}\right)<f\left(\omega_{2}\right)$, entonces pueden descartarse todas las variantes $\omega$, $\omega_{2} \leq \omega$ pues para ellas $f(\alpha)<f(\omega)$.

Demostración:

En efecto, sea que $f\left(\omega_{1}\right)<f\left(\omega_{2}\right), \omega_{2} \leq \omega$. Tomando $\delta=\omega_{2}, \gamma=\omega_{1} \vee \omega_{2}^{*}$, donde $\omega_{2}^{*}$ es el complemento de $\omega_{2}$ respecto de $\omega$; entonces $\delta \vee \gamma=\omega, \delta \wedge \gamma=\omega_{1}$.

Así, por la c-supermodularidad de $f$ se tiene

$$
f(\delta \wedge \gamma)=f\left(\omega_{1}\right)<f\left(\omega_{2}\right)=f(\delta) \quad \Longrightarrow \quad f(\gamma)<f(\delta \vee \gamma)=f(\omega) .
$$

Por lo que $f(\alpha) \leq f(\gamma)<f(\omega)$.

\section{Theorem 4.2 (Segundo principio de descarte).}

Si para $\omega_{1}, \omega_{2} \in \Omega, \omega_{1} \leq \omega_{2}$ se tiene $f\left(\omega_{1}\right)>f\left(\omega_{2}\right)$, entonces pueden descartarse todas las variantes $\omega \leq \omega_{1}$ pues para ellas $f(\alpha)<f(\omega)$.

Demostración: 
En efecto, sea que $f\left(\omega_{1}\right)>f\left(\omega_{2}\right)$ y $\omega \leq \omega_{1}$. Definamos $\delta=\omega_{1}, \gamma=\omega \vee \omega_{1}^{*}$, donde $\omega_{1}^{*}$ es el complemento de $\omega_{1}$ respecto de $\omega_{2}$, entonces $\delta \vee \gamma=\omega_{2}, \delta \wedge \gamma=\omega$.

Así, por propiedad,

$$
f(\delta)=f\left(\omega_{1}\right)>f\left(\omega_{2}\right)=f(\delta \vee \gamma) \quad \Longrightarrow \quad f(\omega)=f(\delta \wedge \gamma)>f(\gamma),
$$

por lo que $f(\omega)>f(\gamma) \geq f(\alpha)$.

\section{Conclusiones.}

1. Los principios de descarte presentados permiten evitar la búsqueda exhaustiva del mínimo de una función c-supermodular definida en la familia de subconjuntos de un conjunto finito dado.

2. Los principios de descarte presentados extienden los resultados de Cherenin y Jachatúrov logrados para funciones supermodulares definidas en la familia de subconjuntos de un conjunto finito dado [8].

3. Los principios de descarte presentados se extienden al caso de una función c-supermodular definida en un retículo finito relativamente complementado.

4. Los principios de descarte presentados permiten evitar la búsqueda exhaustiva del máximo de una función c-supermodular definida en la familia de subconjuntos de un conjunto finito dado.

5. Los principios de descarte presentados extienden los resultados de R.V. Jachatúrov y V.R. Jachatúrov logrados para funciones supermodulares definidas en la familia de subconjuntos de un conjunto finito dado [4] [8].

\section{Agradecimiento.}

Agradezco al Dr. Vladímir Rubénovich Jachatúrov, Jefe del Departmento de Métodos de Sistemas en Desarrollo del Centro de Cálculo de la Academia de Ciencias de Rusia, por su apoyo y asesoramiento en el campo de la optimización combinatoria.

Referencias

[1] Aragonés Salazar, N. O., Minimización de Funciones Supermodulares, Selecciones Matemáticas. Vol 02(02): 49-52 (2015).

[2] Aragonés Salazar, N.O., Minimización de Funciones Supermodulares en un retículo finito relativamente complementado, Selecciones Matemáticas. Vol 04(02): 175-176 (2017).

[3] Grätzer, G., LATTICE THEORY. First concepts and distributive lattices, Dover Publications, INC. Mineola, New York. (1970).

[4] Jachatúrov, R.V., Algoritmos de maximización de funciones supermodulares y su aplicación en la optimización del agrupamiento de zonas de una región, Revista de matemática computacional y física matemática. T.39, Nº1, 33-44 (1999).

[5] Khachaturov, V. R., Lorer, V.E., Investigación y minimización de funciones supermodulares en retículos atómicos // Communications on applied mathematics. Dorodnicyn Computing Centre of RAS, 1987. 41 p.

[6] Jachatúrov, V. R., Métodos matemáticos de programación regional, Nauka, Moscú, Rusia. (1989).

[7] Khachaturov, V. R., Montlevich, V.M., Minimización de funciones supermodulares en retículos distributivos // Communications on applied mathematics. Dorodnicyn Computing Centre of RAS, 1999. 49 p.

[8] Jachatúrov, V. R., Métodos Combinatorios y Algoritmos para la solución de problemas de optimización discreta de gran escala, Nauka, Moscú, Rusia. (2000).

[9] Khachaturov, V.R., Khachaturov, R.V., Supermodular programming on Lattices // Comput. Sci. J. Moldova. 2003. V. 11. No 1(31). P. 43-72.

[10] Mei, J., Zhao, K., Lu, B., Unconstrained Quasi-Submodular Function Optimization, Proceeding AAAI'15 Proceedings of the TwentyNinth AAAI Conference on Artificial Intelligence. (2015) 1191-1197.

[11] Roman, S., Lattices and Ordered Sets. Springer-Verlag, New York, 2008. 